\title{
Cumprimento de expectativas e satisfação com a consulta de Medicina Geral e Familiar
}

Daniel Pinto,* Salomé S. Coutinho,* Carolina Rezende*

\section{RESUMO}

Objectivos: Caracterizar as expectativas dos utentes face à consulta de Medicina Geral e Familiar e identificar a relação entre o seu cumprimento e a satisfação com a consulta.

Tipo de estudo: Observacional, transversal.

Local: Centro de Saúde de Oeiras.

População: Adultos que recorreram à consulta de oito médicos de família.

Métodos: Durante uma semana, os utentes foram seleccionados alternadamente no momento de contacto administrativo e convidados a participar no estudo. Era entregue um questionário pré-consulta, onde o utente deveria assinalar as suas expectativas dentro de dez possíveis e, após a consulta, um questionário de satisfação e um outro identificando os procedimentos efectuados pelo médico.

Resultados: 137 utentes preencheram o questionário inicial e 111 completaram o questionário final. Cada utente tinha, em média, cinco expectativas. Num total de 700 expectativas, $63,1 \%$ foram relativas a obtenção de informação e $36,9 \%$ a alguma acção do médico. As expectativas mais frequentes foram informação sobre o exame objectivo (73,0\%), realização do exame objectivo (68,6\%) e informação sobre o tratamento (67,2\%). A taxa de cumprimento das expectativas foi de $77,2 \%$ e a mediana da satisfação de $83,3 \%$. Encontrou-se uma correlação positiva entre o cumprimento das expectativas e a satisfação com a consulta $(r=, 468, p<0,01)$, que permanece significativa quando ajustada para género e escolaridade. Após o controlo para estes factores, a variância da satisfação explicada pelo cumprimento das expectativas foi de $23,2 \%$.

Conclusões: Os utentes esperam mais frequentemente que o médico realize o exame objectivo e forneça informação acerca do que observou. A maioria das expectativas é cumprida pelo médico, mas quase todos os utentes saem da consulta com pelo menos uma expectativa por cumprir. A satisfação no final da consulta é elevada e correlaciona-se positivamente com o cumprimento das expectativas.

Palavras-chave: Expectativas; Satisfação; Consulta; Cuidados de Saúde Primários.

\section{INTRODUÇÃO}

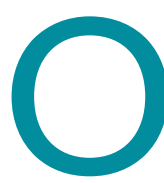

método clínico centrado no paciente defende a concepção da prestação de cuidados de saúde como uma partilha de poder entre o profissional de saúde e o utente, bem como a compreensão da pessoa como um todo e não apenas da doença. ${ }^{1,2} \mathrm{~A}$ abordagem centrada na pessoa associa-se a uma maior satisfação após a consulta ${ }^{2,3}$ e vai de encontro aos desejos do utente. ${ }^{2,4}$ Para este, uma boa comunicação e uma relação de parceria entre

*Médicos Internos de Medicina Geral e Familiar USF S. Julião - Centro de Saúde de Oeiras médico e doente, bem como a promoção da saúde, constituem elementos-chave da consulta ${ }^{4}$ e contribuem para a melhoria do seu estado emocional e físico, para a resolução dos sintomas ${ }^{5}$ e para a adesão a um plano de cuidados. ${ }^{2}$ No entanto, os médicos têm tendência a subvalorizar a importância que a partilha da informação adquire junto dos utentes. ${ }^{6}$

O utente recorre aos serviços de saúde com uma agenda própria, nem sempre coincidente com a do médico, que engloba motivos de consulta muito diversos, relacionados com problemas de saúde (ou outros), preocupações, necessidades e expectativas. ${ }^{7}$ Um dos primeiros passos para o estabelecimento de uma alian- 
ça entre médico e doente é a identificação das expectativas deste face à consulta. Por «expectativa» entende-se aquilo que o utente deseja ou julga que vai acontecer durante a consulta, e é diferente de um "pedido» que é aquilo que o utente explicitamente pede que o médico faça. ${ }^{8,9}$

As expectativas que trazem o utente à consulta são muito diversas e complexas. A sua génese relaciona-se com factores pessoais como a natureza dos sintomas, a percepção da vulnerabilidade à doença, as experiências anteriores e os conhecimentos adquiridos..$^{10} \mathrm{O}$ médico nem sempre identifica correctamente essas expectativas e alguns estudos indicam que 15 a $25 \%$ dos utentes poderão deixar a consulta com alguma expectativa por cumprir., ${ }^{7,11}$ Por outro lado, enquanto muitas vezes os utentes esperam sobretudo que lhes seja dada informação sobre algum assunto, muitos médicos pensam que eles esperam uma acção específica, como o pedido de exames complementares de diagnóstico ou a prescrição de medicamentos. ${ }^{6,11-14}$

O grau de satisfação depende de vários factores, tanto clínicos como não-clínicos, que vão desde o espaço físico onde decorre o encontro até à afabilidade do profissional de saúde e à sua competência técnica tal como é percepcionada pelo utente. ${ }^{6} \mathrm{O}$ médico pode intervir directamente nalguns destes factores, nomeadamente na resposta que proporciona às expectativas do utente.

Em parte, a satisfação do utente depende do cumprimento das expectativas que o trazem à consulta. ${ }^{9,11-15}$ Apesar de nem toda a literatura ser concordante, ${ }^{11,16}$ vários trabalhos mostram que os utentes que vêem as suas expectativas cumpridas saem da consulta mais satisfeitos, o que poderá ter repercussões positivas no seu estado funcional e clínico. ${ }^{11-13,15}$

No entanto, a grande diversidade de metodologias aplicadas e de populações estudadas torna difícil a generalização destas conclusões, havendo ainda um vasto caminho a percorrer na investigação nesta área. Torna-se também importante conhecer a realidade portuguesa, já que diferentes populações parecem valorizar diferentes aspectos dos cuidados de saúde., ${ }^{2,4,17,18}$ Este conhecimento é fundamental para a melhoria dos cuidados de saúde primários numa perspectiva centrada no paciente.

Baseando-se nestes pressupostos, os investigadores conduziram um estudo com o objectivo de caracteri- zar as expectativas dos utentes face à consulta de Medicina Geral e Familiar (MGF) e identificar a relação entre o cumprimento das expectativas do utente e a satisfação com a consulta. Para isso, procurou-se responder às seguintes questões: 1 - Qual a natureza das expectativas dos utentes que recorrem à consulta de MGF e qual a prevalência de cada tipo de expectativa? 2 - Qual a proporção de expectativas cumpridas? 3 - Qual é a satisfação dos utentes em relação à consulta de MGF? 4 - O grau de satisfação aumenta com o cumprimento das expectativas?

\section{MÉTODOS}

\section{Participantes}

Realizou-se um estudo observacional transversal no Centro de Saúde de Oeiras. Foram convidados e aceitaram participar no estudo 8 médicos de família.

Durante uma semana, os utentes que recorreram à consulta destes médicos de família foram seleccionados alternadamente no momento de contacto administrativo e abordados na sala de espera pelos investigadores, que os convidavam a participar no estudo. Foram excluídos os utentes com idade inferior a 18 anos, utentes que não compreendessem a língua portuguesa ou que se mostrassem incapazes de preencher os questionários. Era explicado ao utente o objectivo do estudo, sendo assegurada a confidencialidade da informação prestada e que as suas respostas não seriam reveladas ao respectivo médico de família. Por poder ser um factor de enviesamento dos resultados, decidiu-se omitir desta explicação a hipótese em estudo (a existência de uma correlação positiva entre o cumprimento das expectativas e a satisfação no final da consulta).

\section{Questionários}

Aos utentes que aceitaram responder foram aplicados dois questionários de auto-preenchimento. O primeiro, completado antes da consulta, destinava-se a avaliar as expectativas dos utentes. $\mathrm{O}$ segundo, aplicado após a consulta, avaliava o grau de satisfação do utente com a consulta e a proporção de expectativas cumpridas. Ambos os questionários foram construídos para o estudo e sujeitos a um pré-teste em utentes que não foram incluídos na amostra, o que levou os investigadores a reformular algumas das questões iniciais por dificuldades de interpretação por parte dos utentes. Após a sua correcção, reaplicou-se o questionário noutro 
conjunto de utentes que também não foi incluído no estudo, não se detectando dificuldades no seu preenchimento.

\section{Questionário pré-consulta}

O primeiro questionário destinava-se a caracterizar o utente (idade, género, escolaridade, médico de família e tipo de consulta a que recorria) e a identificar as suas expectativas antes da consulta (Anexo 1). Da classificação desenvolvida por Kravitz e colaboradores, ${ }^{19}$ excluíram-se as expectativas não aplicáveis à organização do sistema de saúde português, adaptando-se as restantes dez frases, que traduzem os tipos de expectativas mais frequentes: observação física, pedido de exames complementares de diagnóstico, referenciação (a outro médico ou a um técnico de saúde), prescrição de novo medicamento, emissão de documento, informação sobre a doença ou sintomas, informação sobre a observação física, informação sobre os resultados dos exames complementares de diagnóstico, informação sobre o tratamento e aconselhamento preventivo.

Para cada afirmação o utente assinalava o seu grau de expectativa de entre 5 respostas possíveis, numa escala de Likert (de «não espero de todo» a "espero muito»). Foram consideradas expectativas aquelas que o utente assinalou como «espero um pouco» ou «espero muito». O questionário foi aplicado na sala de espera, enquanto os utentes aguardavam pela consulta. Se necessário, um dos investigadores podia prestar ajuda no seu preenchimento.

\section{Questionário pós-consulta}

O segundo questionário foi aplicado após a consulta e destinava-se a avaliar o grau de satisfação e o cumprimento das expectativas (Anexo 2).

Para avaliar a satisfação utilizou-se o Consultation Satisfaction Questionnaire desenvolvido por Baker, ${ }^{20}$ adaptado para português. Este questionário avalia três factores de satisfação: aspectos profissionais da consulta, a relação médico-doente e a percepção do tempo de consulta. Consiste em 18 afirmações sobre o médico e a consulta, que são classificadas pelo utente numa escala de Likert. Esta escala é pontuada de um (discordo muito) a cinco (concordo muito), com a pontuação invertida nas afirmações que traduzem insatisfação. $\mathrm{O}$ resultado obtido foi convertido num valor percentual para obter o grau de satisfação do utente, com 100\% a equivaler à satisfação máxima (90 pontos).

Na segunda parte do questionário foi apresentada uma lista de 10 itens, correspondentes às expectativas do primeiro questionário. Foi pedido ao utente que assinalasse as acções ou informações que, segundo a sua perspectiva, tinham sido realizadas pelo médico. Foram consideradas expectativas cumpridas aquelas que foram identificadas no primeiro questionário e assinaladas como realizadas no segundo questionário. Foi também calculada a proporção de expectativas cumpridas dividindo o número de expectativas cumpridas depois da consulta pelo número total de expectativas identificadas antes da consulta.

\section{Análise estatística}

Os registos foram inseridos no programa Microsoft Access 2003 (Microsoft Corp) e a análise estatística foi feita através do SPSS for Windows versão 16 (Chicago, SPSS Inc). A comparação entre grupos foi realizada através do teste T de Student para as variáveis com distribuição normal e do teste de Mann-Whitney para as variáveis com distribuição não normal. O coeficiente de correlação de Pearson foi empregue como medida de associação, utilizando-se a correlação parcial para controlo de factores de confundimento. Estudaram-se como possíveis factores de confundimento do grau de satisfação o género, a idade, a escolaridade, o médico que fez a consulta e o tipo de consulta a que o utente recorreu. Qualquer destes factores era considerado como variável de confundimento se existisse uma correlação com o grau de satisfação após controlo para a proporção de expectativas cumpridas. Para todos os testes foi definido um nível de significância de $5 \%$.

\section{RESULTADOS}

Foram convidados a participar no estudo 155 utentes, 14 recusaram. A maioria apontou a falta de tempo como razão para a recusa, os restantes não forneceram qualquer justificação. Um dos utentes abandonou o estudo e não entregou o questionário pré-consulta, dois foram excluídos por preenchimento do questionário por terceiros e um por incapacidade para preencher o questionário correctamente. Assim, foram obtidos 137 questionários de expectativas válidos antes da consulta. A idade média dos utentes da amostra foi de 54,8 anos e 


QUADRO I. Características dos participantes $(\mathrm{n}=137)$
\begin{tabular}{l|r} 
Idade média & 54,8 anos \\
(desvio padrão) & $( \pm 18,1)$ \\
\hline Género $n(\%)$ & \\
$\quad$ Mulheres & $91(66,4)$ \\
Homens & $46(33,6)$ \\
\hline Escolaridade $n(\%)$ & \\
$\quad$ Desconhecida & $3(2,2)$ \\
Nenhuma & $3(2,2)$ \\
Primária & $32(23,4)$ \\
Básica & $28(20,4)$ \\
Secundária & $35(25,5)$ \\
$\quad$ Superior & $36(26,5)$ \\
\hline Tipo de consulta n (\%) & \\
$\quad$ Consulta do dia & $61(44,5)$ \\
Adultos & $71(51,8)$ \\
Planeamento familiar/rastreio oncológico & $4(2,9)$ \\
Saúde materna & $1(0,7)$
\end{tabular}

66,4\% era do género feminino (Quadro I).

Onze utentes abandonaram o centro de saúde sem preencher o questionário pós-consulta. A maioria não indicou as razões do abandono, os restantes invocaram falta de tempo. Os utentes que não completaram o segundo questionário eram em média 12,7 anos mais novos que aqueles que o fizeram ( $\mathrm{p}=0,026)$, não diferindo significativamente quanto a género, escolaridade e tipo de consulta a que recorreram. Foram excluídos da análise de correlação 15 utentes por apresentarem incorrecções no preenchimento do questionário de satisfação. Desta forma, a análise do cumprimento das expectativas e da satisfação fez-se apenas em 111 utentes.

\section{Expectativas antes da consulta}

$\mathrm{O}$ intervalo de expectativas assinaladas variou entre 0 e 10, com uma média de 5,11 expectativas por utente. Foram identificadas 700 expectativas, das quais 442 (63,1\%) estavam relacionadas com a obtenção de informação e 258 (36,9\%) com acções específicas por parte do médico. $97,1 \%$ dos utentes tinha pelo menos uma expectativa antes da consulta (quatro utentes não assinalaram qualquer expectativa), 93,4\% esperava algum tipo de acção e 89,1\% esperava algum tipo de informação. O Quadro II mostra a prevalência de cada tipo de ex-

\begin{tabular}{|c|c|c|}
\hline \multicolumn{3}{|c|}{$\begin{array}{l}\text { QUADRO II. Proporção dos utentes que assinalou cada } \\
\text { tipo de expectativa }(n=137)\end{array}$} \\
\hline \multicolumn{2}{|l|}{ Expectativa } & $\begin{array}{c}\% \\
\text { utentes }\end{array}$ \\
\hline \multirow{5}{*}{ Acção } & Observação física & 68,6 \\
\hline & $\begin{array}{l}\text { Pedido de exames complementares } \\
\text { de diagnóstico }\end{array}$ & 49,6 \\
\hline & $\begin{array}{l}\text { Referenciação (a outro médico } \\
\text { ou a um técnico de saúde) }\end{array}$ & 24,8 \\
\hline & Prescrição de novo medicamento & 23,4 \\
\hline & Emissão de documento & 21,9 \\
\hline \multirow{5}{*}{ Informação } & $\begin{array}{l}\text { Informação sobre a doença ou } \\
\text { sintomas }\end{array}$ & 66,4 \\
\hline & Informação sobre a observação física & 73,0 \\
\hline & $\begin{array}{l}\text { Informação sobre os resultados } \\
\text { dos exames complementares de } \\
\text { diagnóstico }\end{array}$ & 50,4 \\
\hline & Informação sobre o tratamento & 67,2 \\
\hline & Aconselhamento preventivo & 65,7 \\
\hline
\end{tabular}

pectativa na amostra. As expectativas mais frequentes foram o fornecimento de informação sobre a observação e a realização do exame objectivo. As expectativas foram semelhantes entre os utentes que iriam ter uma consulta programada (saúde do adulto) ou uma consulta não programada (consulta do dia) (Quadro III).

\section{Cumprimento das expectativas}

A taxa de cumprimento das expectativas foi de $77,2 \%$. Para a maioria das expectativas a taxa de cumprimento foi superior a $80 \%$, sendo mais frequentemente correspondidas as expectativas de pedido de exames complementares de diagnóstico, informação sobre o tratamento ou medicação e emissão de um documento administrativo (Quadro IV). As expectativas menos cumpridas foram a prescrição de um novo medicamento e a referenciação. Quatro utentes viram todas as suas expectativas cumpridas e dois não assinalaram qualquer expectativa. Em 111 utentes, 105 (94,6\%) ficaram com pelo menos uma expectativa por cumprir.

\section{Satisfação no final da consulta}

A satisfação com a consulta variou entre 38,9 e 100\%, com um valor mediano de $83,3 \%$ (desvio padrão $12,2 \%$ ). 
QUADRO III. Prevalência de expectativas por tipo de consulta (consulta do dia $-\mathbf{n}=61$; saúde de adultos $-\mathbf{n}=71$ ).

\section{Expectativa}

Observação física

Pedido de exames complementares de diagnóstico

Referenciação (a outro médico ou a um técnico de saúde)

Prescrição de novo medicamento

Emissão de documento

Informação sobre a doença ou sintomas

Informação sobre a observação física

Informação sobre os resultados dos exames complementares de diagnóstico

Informação sobre o tratamento

Aconselhamento preventivo

Consulta do dia (\%)

\begin{tabular}{l|l}
\hline 67,2 & 69,0 \\
\hline 49,2 & 50,7 \\
\hline 34,4 & 16,9 \\
\hline 27,9 & 21,1 \\
\hline 32,9 & 12,7 \\
\hline 70,5 & 66,2 \\
\hline 77,0 & 70,4 \\
\hline 49,2 & 50,7 \\
\hline 72,1 & 64,8 \\
\hline 60,7 & 69,0 \\
\hline
\end{tabular}

Após controlo para a proporção de expectativas cumpridas, foi encontrada uma associação entre o grau de satisfação e o género ( $\mathrm{r}=, 199 \mathrm{p}=0,04)$, com as mulheres a referirem maior satisfação que os homens $(82,4 \%$ vs $78,7 \%)$, e a escolaridade ( $\mathrm{r}=-, 241 \mathrm{p}=0,01)$, tendo os utentes referido menor satisfação à medida que a escolaridade aumentava (de $87,0 \%$ nos utentes analfabetos a $76,7 \%$ nos que completaram o ensino superior). Não houve associação com a idade, o médico que fez a consulta e o tipo de consulta. Assim, género e escolaridade foram considerados como factores de confundimen- to da associação entre satisfação e expectativas cumpridas.

Associação cumprimento das expectativas - satisfação Encontrou-se uma correlação positiva e estatisticamente significativa entre o cumprimento das expectativas do utente e o grau de satisfação no final da consulta $\mathrm{r}=, 468(\mathrm{p}<0,001)$ (Figura 1). Esta associação permaneceu significativa na correlação parcial com controlo para o género e a escolaridade dos utentes $-r=, 482$ $(p<0,001)$. A variância da satisfação explicada pelo cum-

\begin{tabular}{|c|c|c|c|c|}
\hline Expectativa & $\begin{array}{l}\text { Cumprida } \\
\text { (n) }\end{array}$ & $\begin{array}{c}\text { Não } \\
\text { cumprida } \\
\text { (n) }\end{array}$ & $\begin{array}{c}\text { Taxa } \\
\text { cumprimento } \\
(\%)\end{array}$ & $\begin{array}{c}\text { Não } \\
\text { assinalada } \\
\text { (n) }\end{array}$ \\
\hline Observação física & 64 & 13 & 83,1 & 34 \\
\hline Pedido de exames complementares de diagnóstico & 51 & 9 & 85,0 & 51 \\
\hline Referenciação (a outro médico ou a um técnico de saúde) & 13 & 17 & 43,3 & 81 \\
\hline Prescrição de novo medicamento & 12 & 16 & 42,9 & 83 \\
\hline Emissão de documento & 20 & 4 & 83,3 & 87 \\
\hline Informação sobre a doença ou sintomas & 64 & 14 & 82,1 & 33 \\
\hline Informação sobre a observação física & 65 & 18 & 78,3 & 28 \\
\hline $\begin{array}{l}\text { Informação sobre os resultados dos exames complementares } \\
\text { de diagnóstico }\end{array}$ & 49 & 10 & 83,1 & 52 \\
\hline Informação sobre o tratamento & 65 & 12 & 84,4 & 34 \\
\hline Aconselhamento preventivo & 53 & 22 & 70,7 & 36 \\
\hline Total & 456 & 135 & 77,2 & $2 *$ \\
\hline
\end{tabular}

* Número de utentes que não assinalou qualquer expectativa. 


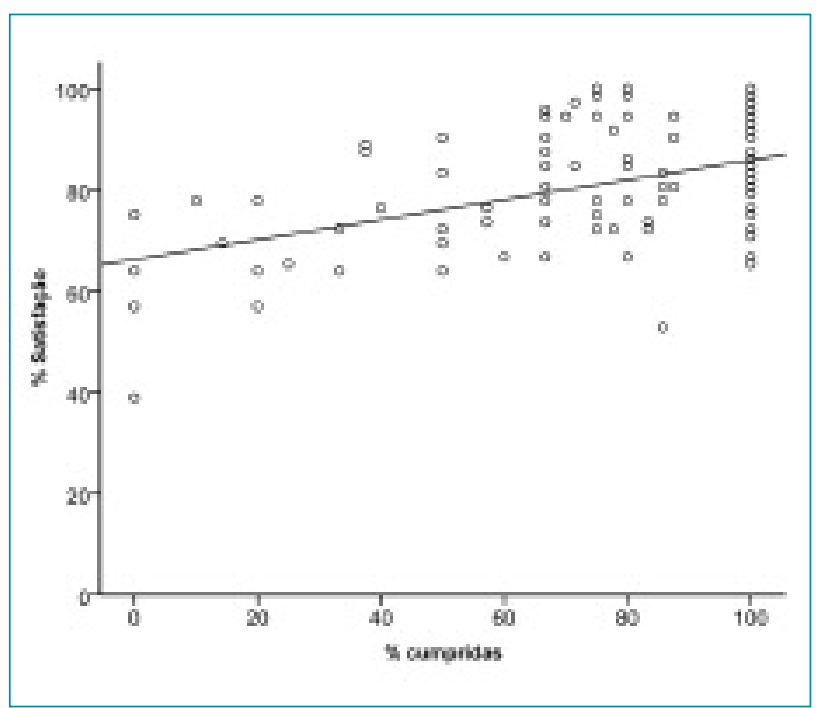

Figura 1. Gráfico de dispersão da satisfação em função da proporção de expectativas cumpridas, com linha de regressão linear - $r=, 468(n=111)$.

primento das expectativas, após o controlo para género e escolaridade, foi de $23,2 \%\left(r^{2}=0,232\right)$.

Foram também analisadas as expectativas individualmente para identificar aquelas cujo cumprimento influenciava o grau de satisfação (QuadroV). Verificou-se que os utentes em quem as expectativas de pedido de exames complementares de diagnóstico, informação sobre a doença ou sintomas, informação sobre a observação física, informação sobre os resultados dos exames complementares de diagnóstico e aconselhamento preventivo foram cumpridas pelo médico estavam em média mais satisfeitos que aqueles em quem essas expectativas não foram cumpridas.

\section{DISCUSSÃO}

Este estudo procurou, em primeiro lugar, caracterizar as expectativas dos utentes face às consultas de Medicina Geral e Familiar. Quase todos os utentes apresentaram pelo menos uma expectativa antes da consulta, tendo assinalado em média cinco em dez possíveis. A comparação destes resultados com os de outros estudos é difícil devido, por um lado, à falta de consenso acerca da nomenclatura das expectativas e, por outro, à grande variabilidade dos métodos utilizados para as avaliar. O uso de questionários tende a sobreavaliar o número de expectativas assinaladas. ${ }^{9,11,13}$ Kravitz $^{9}$ con- cluiu que os utentes a quem foi administrado um questionário indicaram maior número de expectativas (12 em 28 possíveis) do que aqueles a quem foi feita uma entrevista (três, sem número máximo de expectativas definido) e tiveram um número maior de expectativas não cumpridas. Ainda assim, estas diferenças não influenciaram a relação com a satisfação no final da consulta. Os nossos resultados são semelhantes aos obtidos noutros trabalhos que mediram as expectativas por questionário, em que o número médio de expectativas assinaladas pelos utentes foi cerca de metade do total constante no questionário..$^{9,12-14}$

A maior parte das expectativas dos utentes enquadra-se nos pedidos de informação, o que está de acordo com outros estudos. ${ }^{11-14,19}$ Tem sido descrito que os médicos identificam acções específicas como principais expectativas dos utentes. ${ }^{11}$ Contrariamente a esta percepção as expectativas de emissão de documentos, prescrição de novos medicamentos e referenciação foram as menos frequentes.

Foi sugerido que as expectativas entre os utentes que se apresentam com sintomas específicos são diferentes das expectativas apresentadas em consultas de rotina. ${ }^{10,12,14}$ Considerando que as consultas do dia seriam mais motivadas por queixas específicas que as consultas programadas, seria de esperar que as expectativas nos dois grupos fossem diferentes. Contudo, os nossos resultados não evidenciaram diferenças entre os utentes que vinham para consulta do dia e aqueles que vinham para consulta programada. Optámos por não fazer testes estatísticos para avaliar estas diferenças porque esse não era um dos objectivos do nosso estudo e, pelo elevado número de testes a realizar, poderíamos incorrer num erro do tipo alfa.

Os médicos cumpriram a maioria das expectativas, mas, apesar disso, quase todos os utentes ficaram com alguma expectativa por cumprir. Esta proporção é superior à encontrada noutros estudos ${ }^{10,15}$ e poderá estar relacionada com o instrumento de medida.

Apesar das expectativas de informação serem as mais frequentes, das quatro mais cumpridas, três são relativas a acções do médico e apenas uma relativa a pedidos de informação. Tem sido proposto que os médicos tendem a subvalorizar as expectativas de informação, ${ }^{11,12}$ o que poderá limitar a sua capacidade para as identificar e, consequentemente, cumprir. Parece-nos 
QUADRO V. Satisfação média em função do cumprimento de cada expectativa.

\begin{tabular}{|c|c|c|c|}
\hline \multirow[b]{2}{*}{ Expectativa } & \multicolumn{2}{|c|}{ Satisfação (\%) se expectativa } & \multirow[b]{2}{*}{$\mathbf{p}$} \\
\hline & Cumprida & Não cumprida & \\
\hline Observação física (n = 77) & 84,5 & 78,6 & $0,073 *$ \\
\hline Pedido de exames complementares de diagnóstico $(n=60)$ & 83,5 & 73,5 & $0,023 *$ \\
\hline Referenciação (a outro médico ou a um técnico de saúde) $(n=30)$ & 80,3 & 83,1 & $0,488 \dagger$ \\
\hline Prescrição de novo medicamento $(n=28)$ & 82,8 & 78,5 & $0,184 \dagger$ \\
\hline Emissão de documento $(n=24)$ & 78,4 & 79,2 & $0,926 *$ \\
\hline Informação sobre a doença ou sintomas $(n=78)$ & 83,8 & 73,9 & $0,002 \dagger$ \\
\hline Informação sobre a observação física $(\mathrm{n}=83)$ & 84,6 & 78,5 & $0,035^{*}$ \\
\hline Informação sobre os resultados dos exames complementares de diagnóstico ( $n=59$ ) & 86,4 & 78,5 & $0,018 \dagger$ \\
\hline Informação sobre o tratamento $(n=77)$ & 82,6 & 76,5 & $0,071^{*}$ \\
\hline Aconselhamento preventivo $(n=75)$ & 85,5 & 76,4 & $<0,001^{*}$ \\
\hline
\end{tabular}

* Teste T de Student. † Teste de Mann-Whitney

mais provável que os médicos não cumpram as expectativas de informação por não as identificarem e não por optarem conscientemente por não dar ao utente a informação que este pretende.

Entre as expectativas cujo cumprimento pode ser medido directamente nos registos clínicos (pedido de exames complementares, referenciação e medicação) e, por isso, mais estudadas na literatura, os nossos resultados diferem um pouco do que tem sido descrito. ${ }^{12,13,16} \mathrm{O}$ cumprimento da expectativa de pedido de exames complementares foi maior na nossa amostra, enquanto a prescrição de novos medicamentos foi menor. Isto poderá estar relacionado com o facto terem sido incluídas consultas de seguimento de doença crónica, em que a realização periódica de exames complementares está frequentemente indicada e a medicação está estabilizada. Outros autores têm estudado utentes que recorrem ao médico por doença aguda, ${ }^{12,13}$ o que poderá explicar as diferenças encontradas. A referenciação, à semelhança de outros estudos, foi a expectativa menos cumprida.

Têm sido levantadas algumas questões relativamente à metodologia utilizada para avaliação do cumprimento das expectativas através de questionários pré- $\mathrm{e}$ pós-consulta. ${ }^{9,16}$ Em primeiro lugar, este método não permite avaliar quais as expectativas verbalizadas na forma de pedidos durante a consulta. Em segundo lugar, também não permite avaliar o papel da negociação por parte do médico durante a consulta, que poderá al- terar as expectativas do utente. Finalmente, este é um método subjectivo que se baseia naquilo que, na perspectiva do utente, foi ou não feito durante a consulta. Porém, a investigação tem mostrado que médico e utente nem sempre estão de acordo quanto ao que foi feito na consulta, sobretudo no que diz respeito a informação dada. Jackson e Kroenke, ${ }^{12}$ num estudo sobre o efeito das expectativas não cumpridas em doentes com sintomas físicos, concluíram que a satisfação e outros outcomes melhoravam em doentes que reportavam ter recebido informação diagnóstica ou prognóstica do seu médico. A mesma associação não se verificou quando a partilha dessa informação era reportada pelo médico, reflectindo que esta nem sempre se torna perceptível para o utente. Considerando que o endpoint no nosso estudo é a satisfação do utente, é provável que a sua perspectiva seja o parâmetro mais relevante para este resultado.

Na nossa amostra encontrou-se uma correlação positiva entre o cumprimento das expectativas e a satisfação no final da consulta, que permite explicar $23,2 \%$ da variabilidade na satisfação. Isto implica que os restantes $76,8 \%$ da variabilidade serão explicados por outros factores não medidos no nosso estudo. Dois destes foram identificados por nós como factores de confundimento: o género e a escolaridade do utente. Na literatura são descritos diversos outros factores que podem influenciar a satisfação. ${ }^{6,9,14}$ Alguns ligados ao próprio utente (além do género e da escolaridade, a 
idade, a raça e a auto-percepção do estado de saúde), outros ao médico (competência clínica e interpessoal, envolvimento do utente), outros ainda às condições e organização dos serviços de saúde (ambiente físico do consultório, pessoal administrativo, sistema de marcação de consultas, tempo de espera, aspectos financeiros, transportes e estacionamento). Destes, muitos são inalteráveis, nomeadamente os que se relacionam com o próprio utente, e outros não estão directamente dependentes do médico, como os relacionados com a organização dos serviços. Assim, embora a contribuição do cumprimento das expectativas do utente para a satisfação final possa à primeira vista ser modesta, este factor torna-se mais relevante por ser um em cuja melhoria o médico pode intervir directamente. Para além disso, apesar de globalmente terem um peso importante na satisfação, o peso individual de cada um dos outros factores permanece por determinar e é provável que poucos sejam tão importantes quanto o cumprimento das expectativas.

A investigação prévia tem sugerido que diferentes expectativas possam ter um impacto diferente na satisfação final quando cumpridas ou não. ${ }^{12,14,21}$ Quando comparamos os níveis de satisfação entre os utentes para os quais uma determinada expectativa foi ou não cumprida, as expectativas de informação parecem ter um peso maior na satisfação final. Ao contrário de outros estudos, na nossa amostra a diferença de satisfação entre os utentes em que a expectativa de pedido de exames complementares foi cumprida e aqueles em que não foi, não só foi estatisticamente significativa, como foi a maior observada em termos absolutos. Estes resultados devem, contudo, ser interpretados com cuidado; não só porque o número reduzido de participantes em cada grupo pode não permitir distinguir o efeito do cumprimento da expectativa, mas também porque não foi feita qualquer correcção para múltiplos testes estatísticos, aumentando a probabilidade de erro tipo alfa.

O nosso estudo tem algumas limitações metodológicas que importa discutir. Em primeiro lugar, o processo de amostragem utilizado corre algum risco de induzir um viés de selecção. Apesar disso, a distribuição entre géneros e a média de idades na nossa amostra é semelhante à encontrada noutro estudo nacional em utilizadores dos centros de saúde. ${ }^{22} \mathrm{O}$ nível de escola- ridade na nossa amostra foi superior ao encontrado neste estudo. Tal poderá dever-se ao nível de escolaridade no Concelho de Oeiras ser superior à média nacional. ${ }^{23}$

Segundo, os questionários utilizados não foram previamente validados. A taxonomia de classificação das expectativas não é consensual na literatura. Baseámonos na descrição feita por Kravitz, ${ }^{19}$ que estudou utentes dos Estados Unidos da América. É possível que existam diferenças culturais importantes e que algumas expectativas dos portugueses não constassem do nosso questionário (talvez seja essa a razão porque quatro utentes não assinalaram qualquer expectativa). O pré-teste realizado permitiu confirmar que o questionário era entendido pelos utentes, mas este teste foi feito a um grupo demasiado pequeno de utentes para detectar expectativas pouco frequentes que não tivessem sido incluídas. Também o questionário de satisfação foi traduzido e adaptado da versão original em inglês e não estava validado para a população portuguesa. Existem alguns instrumentos de avaliação da satisfação de utentes de cuidados de saúde primários validados para Portugal, mas nenhum deles avalia especificamente a satisfação com uma consulta, pelo que eram inadequados para o nosso estudo. Apesar de não ter sido feita uma validação formal, o pré-teste permitiu mais uma vez corrigir alguns aspectos da tradução e não foram encontradas dificuldades na interpretação da versão final utilizada no estudo.

Será útil, de futuro, desenvolver um método de avaliação de expectativas fiável e bem aceite que permita a comparação entre diferentes estudos, à semelhança do que já foi feito para os pedidos durante a consulta (TORP).${ }^{19}$ Em Portugal é importante começar por realizar estudos de índole qualitativa que sirvam de base à construção de um questionário para medir expectativas. Estes permitiriam verificar se existem, de facto, diferenças culturais entre as expectativas face à consulta de MGF apresentadas pelos portugueses ou por outras populações e também entre diferentes grupos étnicos dentro do país.

É também importante aprofundar a investigação sobre outros factores modificáveis que podem influenciar a satisfação dos utentes e clarificar a associação entre a satisfação com a consulta e os outcomes relacionados com a saúde. A investigação nesta área está ainda pou- 
co desenvolvida e tem apresentado resultados nem sempre concordantes. É necessário comprovar que uma intervenção para melhorar a exploração das expectativas do utente na consulta se traduz em ganhos de saúde, para que possam ser recomendadas acções visando aprofundar esta competência por parte dos médicos de família.

Em conclusão, o nosso estudo traz novos conhecimentos sobre as expectativas dos utentes face à consulta de Medicina Geral e Familiar em Portugal. Os utentes esperam mais frequentemente que o médico realize o exame objectivo e forneça informação acerca do que observou. A maioria das expectativas é cumprida pelo médico, mas quase todos os utentes saem da consulta com pelo menos uma expectativa por cumprir. A satisfação no final da consulta é elevada e correlaciona-se positivamente com o cumprimento das expectativas.

\section{AGRADECIMENTOS}

Aos Drs. José Mendes Nunes, Teresa Libório e Áurea Farinha, nossos orientadores de formação, pelo seu apoio e incentivo durante a realização deste trabalho e pelos seus comentários construtivos ao manuscrito.

À Dra. Inês Gomes pelas correcções ao resumo em inglês.

\section{REFERÊNCIAS}

1. McWhinney IR.A textbook of Family Medicine. 2nd edition. Oxford: Oxford University Press; 1997.

2. Irwin RS, Richardson ND. Patient-focused care: using the right tools. Chest 2006 Jul; 130 (Suppl 1): S73-82.

3. Kinnersley P, Stott N, Peters TJ, Harvey I. The patient-centredness of consultations and outcome in primary care. Br J Gen Pract 1999 Sep; 49 (446): 711-6.

4. Little P, Everitt H, Williamson I, Warner G, Moore M, Gould C, et al. Preferences of patients for patient centred approach to consultation in primary care: observational study. BMJ. 2001 Feb 24;322 (7284): 468-72.

5. Stewart MA. Effective physician-patient communication and health outcomes: a review. CMAJ 1995 May 1; 152 (9): 1423-33.

6. Laine C, Davidoff F, Lewis CE, Nelson EC, Nelson E, Kessler RC, et al. Important elements of outpatient care: a comparison of patients' and physicians' opinions. Ann Intern Med 1996 Oct 15; 125 (8): 640-5.

7. Kroenke K. Patient expectations for care: how hidden is the agenda? Mayo Clin Proc 1998 Feb; 73 (2): 191-3.

8. Kravitz RL. Measuring patients' expectations and requests. Ann Intern Med 2001 May 1; 134 (9 Pt 2): 881-8.

9. Kravitz RL, Callahan EJ, Azari R, Antonius D, Lewis CE. Assessing patients' expectations in ambulatory medical practice. Does the measurement approach make a difference? J Gen Intern Med 1997 Jan; 12 (1): 67-72.

10. Kravitz RL, Callahan EJ, Paterniti D, Antonius D, Dunham M, Lewis CE. Prevalence and sources of patients' unmet expectations for care. Ann Intern Med 1996 Nov 1; 125 (9): 730-7.

11. Rao JK, Weinberger M, Kroenke K. Visit-specific expectations and pa- tient-centered outcomes: a literature review. Arch Fam Med 2000 NovDec; 9 (10): 1148-55.

12. Jackson JL, Kroenke K. The effect of unmet expectations among adults presenting with physical symptoms. Ann Intern Med 2001 May 1; 134 (9 Pt 2): 889-97.

13. Ruiz-Moral R, Pérula de Torres LA, Jaramillo-Martin I. The effect of patients' met expectations on consultation outcomes: a study with family medicine residents. J Gen Intern Med 2007 Jan; 22 (1): 86-91.

14. Joos SK, Hickam DH, Borders LM. Patients' desires and satisfaction in general medicine clinics. Public Health Rep 1993 Nov-Dec; 108 (6): 751-9.

15. Bell RA, Kravitz RL, Thom D, Krupat E, Azari R. Unmet expectations for care and the patient-physician relationship. J Gen Intern Med 2002 Nov; 17 (11): 817-24.

16. Peck BM, Ubel PA, Roter DL, Goold SD, Asch DA, Jeffreys AS, et al. Do unmet expectations for specific tests, referrals, and new medications reduce patients' satisfaction? J Gen Intern Med 2004 Nov; 19 (11): 1080-7.

17. Krupat E, Bell RA, Kravitz RL, Thom D, Azari R. When physicians and patients think alike: patient-centered beliefs and their impact on satisfaction and trust. J Fam Pract 2001 Dec; 50 (12): 1057-62.

18. Zemencuk JK, Feightner JW, Hayward RA, Skarupski KA, Katz SJ. Patients' desires and expectations for medical care in primary care clinics. J Gen Intern Med 1998 Apr; 13 (4): 273-6.

19. Kravitz RL, Bell RA, Franz CE. A taxonomy of requests by patients (TORP): a new system for understanding clinical negotiation in office practice. J Fam Pract 1999 Nov; 48 (11): 872-8.

20. Baker R. Development of a questionnaire to assess patients' satisfaction with consultations in general practice. Br J Gen Pract 1990 Dec; 40 (341): 487-90.

21. Zebiene E, Razgauskas E, Basys V, Baubiniene A, Gurevicius R, Padaiga $Z$, et al. Meeting patient's expectations in primary care consultations in Lithuania. Int J Qual Health Care 2004 Feb;16 (1): 83-9.

22. Ferreira PL, Raposo V, Godinho P. A voz dos utilizadores dos Centros de Saúde - europep 2003/2004 [Internet]. Coimbra: Centro de Estudos e Investigação em Saúde da Universidade de Coimbra; 2005. Disponível em: http://europep.fe.uc.pt/reports/free/0000.pdf [acedido a 04/02/2009].

23. Oeiras Factos e Números, Câmara Municipal de Oeiras, 2003. Diponível em: http://www.cmoeiras.pt/Ciberstore_backoffice/output_ efile.aspx?sid=7333e 774-a6eb-4a30-918aebe834e15f0a\&cntx =mF4LWJiukXUrOZAJeF72tsL\%2B7adYGTNCw7X9Pw\%2FwxithJdFjebdEJIW6Se8ufYPKX2ib0PGOo3kpZAd7NZI\%2Brw\%3D\%3D\&idf=6 64 [acedido a 06/02/2009].

Os autores declararam não possuir conflitos de interesses

\section{ENDEREÇO PARA CORRESPONDÊNCIA}

Daniel Pinto

Rua da Fonte, 7 - Tornada

2500-315 Caldas da Rainha

Email: danieljllpinto@gmail.com

Recebido em 02/03/2009

Aceite para publicação em 15/07/2009 


\section{ABSTRACT}

\section{MEETING EXPECTATIONS AND PATIENT SATISFACTION WITH FAMILY MEDICINE OFFICE VISITS}

Objectives: To characterize patient expectations in Family Medicine visits, and to identify the relationship between meeting them and the satisfaction at the end of the visit.

Design: Observational, longitudinal.

Setting: Oeiras Health Center.

Patients: Adults who visited eight family doctors.

Methods: During one week, patients were alternately selected when they contacted the secretary and invited to participate. A pre-office visit questionnaire was given, where patients were asked to select their expectations from a list of ten possibilities. After the visit, patients filled out a satisfaction questionnaire and another identifying what had been done by the doctor.

Results: 137 patients completed the initial questionnaire and 111 completed the final questionnaire. Each patient reported, on average, five expectations. From a total of 700 expectations, $63.1 \%$ were related to obtaining information, and $36.9 \%$ to the physician's actions. The most frequent expectations dealt with information about the physical examination (73.0\%), having a physical examination (68.6\%), and information about treatment $(67.2 \%)$. The rate of expectations met was $77.2 \%$ and median satisfaction was $83.3 \%$. We found a positive correlation between meeting expectations and office visit satisfaction ( $r=.468$, $\mathrm{p}<0.01$ ), which remained significant after adjustment for sex and educational status. After controlling for these factors, the variance in satisfaction explained by met expectations was $23.2 \%$.

Conclusions: Patients most often expect doctors to perform a physical examination and give them information about what they find. Most expectations are met by doctors, but almost all patients leave with at least one unmet expectation. Satisfaction at the end of the visit is high and positively correlates with the meeting of their expectations.

Keywords: Expectations; Patient Satisfaction; Office Visits; Primary Health Care. 


\section{Dados Gerais}

\begin{tabular}{|c|c|c|}
\hline \multicolumn{3}{|l|}{ Idade __ anos } \\
\hline \multicolumn{3}{|l|}{ Sexo $M \square F \square$} \\
\hline \multicolumn{3}{|l|}{ Escolaridade } \\
\hline \multicolumn{3}{|l|}{ Médico de Família } \\
\hline \multirow[t]{4}{*}{ Tipo de consulta } & $\square$ & Consulta do dia (marcada hoje) \\
\hline & $\square$ & Adultos (marcada com antecedência \\
\hline & & Grávidas (Saúde Materna) \\
\hline & & Planeamento familiar \\
\hline
\end{tabular}

\section{QUESTIONÁRIO}

No verso da folha encontra algumas perguntas acerca do que espera da consulta que vai ter. Pedimos que nos diga o que espera ou gostaria que acontecesse na sua consulta de hoje e não o que acha importante nas consultas em geral ou o que espera numa das próximas consultas.

Em relação a cada uma das perguntas existem cinco hipóteses de resposta, que vão desde não espero de todo até espero muito. Deve assinalar aquela que lhe parece mais adequada de acordo com o que espera para a consulta de hoje.

\begin{tabular}{|c|c|c|c|c|c|}
\hline Nesta consulta eu espero que o meu médico... & $\begin{array}{c}\text { Não espero } \\
\text { de todo }\end{array}$ & $\begin{array}{c}\text { Não } \\
\text { espero }\end{array}$ & $\begin{array}{l}\text { Não } \\
\text { sei }\end{array}$ & $\begin{array}{c}\text { Espero } \\
\text { um pouco }\end{array}$ & $\begin{array}{l}\text { Espero } \\
\text { muito }\end{array}$ \\
\hline $\begin{array}{l}1 \text {.... me faça uma observação física (por exemplo: auscultar, } \\
\text { ver a garganta, palpar a barriga, medir a tensão arterial, etc.). }\end{array}$ & 1 & 2 & 3 & 4 & 5 \\
\hline 2 ... me peça exames (análises, radiografias, electrocardiograma, etc.). & 1 & 2 & 3 & 4 & 5 \\
\hline $\begin{array}{l}3 \text {... me envie para uma consulta de outra especialidade } \\
\text { (cirurgia, cardiologia, fisioterapia, etc.). }\end{array}$ & 1 & 2 & 3 & 4 & 5 \\
\hline $\begin{array}{l}4 \text {... me passe a receita para um novo medicamento } \\
\text { (medicamento que não tomava habitualmente). }\end{array}$ & 1 & 2 & 3 & 4 & 5 \\
\hline 5 ... me passe um papel / documento (atestado, baixa, declaração, etc.) & 1 & 2 & 3 & 4 & 5 \\
\hline 6 ... me explique a minha doença / as minhas queixas. & 1 & 2 & 3 & 4 & 5 \\
\hline $\begin{array}{l}7 \text {... me explique o que observou em mim (auscultação, garganta, } \\
\text { barriga, tensão arterial, etc.). }\end{array}$ & 1 & 2 & 3 & 4 & 5 \\
\hline 8 ... me explique os resultados dos exames. & 1 & 2 & 3 & 4 & 5 \\
\hline $\begin{array}{l}9 \text {... me explique como vai ser o meu tratamento (como tomar os } \\
\text { medicamentos, outros tratamentos, consultas de seguimento, etc.). }\end{array}$ & 1 & 2 & 3 & 4 & 5 \\
\hline $\begin{array}{l}10 \text {... me dê conselhos sobre como viver saudavelmente } \\
\text { (alimentação, exercício, stress, etc.). }\end{array}$ & 1 & 2 & 3 & 4 & 5 \\
\hline
\end{tabular}

Não se esqueça que, no final da consulta, gostaríamos que respondesse a mais algumas perguntas rápidas. Obrigado pela sua colaboração. 


\section{ANEXO 2}

\section{QUESTIONÁRIO}

Pedimos-lhe agora que nos responda a algumas perguntas sobre a forma como decorreu a sua consulta de hoje.

Em relação a cada uma das perguntas existem cinco hipóteses de resposta, que vão desde discordo completamente até concordo completamente. Deve assinalar aquela que lhe parece mais adequada de acordo com a consulta de hoje.

\begin{tabular}{|c|c|c|c|c|c|}
\hline & $\begin{array}{c}\text { Discordo } \\
\text { muito } \\
\end{array}$ & Discordo & $\begin{array}{l}\text { Assim- } \\
\text {-assim }\end{array}$ & Concordo & $\begin{array}{c}\text { Concordo } \\
\text { muito } \\
\end{array}$ \\
\hline 1. Fiquei totalmente satisfeito com esta consulta. & $\square$ & $\square$ & $\square$ & $\square$ & $\square$ \\
\hline 2. O médico fez-me uma observação muito completa e cuidadosa. & $\square$ & $\square$ & $\square$ & $\square$ & $\square$ \\
\hline 3. Vou seguir o conselho do médico porque é absolutamente correcto. & $\square$ & $\square$ & $\square$ & $\square$ & $\square$ \\
\hline 4. Senti-me à vontade para contar ao médico aspectos muito pessoais. & $\square$ & $\square$ & $\square$ & $\square$ & $\square$ \\
\hline 5. O tempo de consulta foi um pouco curto. & $\square$ & $\square$ & $\square$ & $\square$ & $\square$ \\
\hline 6. O médico informou-me totalmente acerca do meu tratamento. & $\square$ & $\square$ & $\square$ & $\square$ & $\square$ \\
\hline 7. Alguns aspectos desta consulta poderiam ter sido melhores. & $\square$ & $\square$ & $\square$ & $\square$ & $\square$ \\
\hline 8. Este médico desconhece várias coisas a meu respeito. & $\square$ & $\square$ & $\square$ & $\square$ & $\square$ \\
\hline 9. O médico observou-me cuidadosamente. & $\square$ & $\square$ & $\square$ & $\square$ & $\square$ \\
\hline 10. O médico encarou-me como ser humano. & $\square$ & $\square$ & $\square$ & $\square$ & $\square$ \\
\hline $\begin{array}{l}\text { 11. O tempo de consulta não foi suficiente para contar todos os } \\
\text { problemas que tinha. }\end{array}$ & $\square$ & $\square$ & $\square$ & $\square$ & $\square$ \\
\hline 12. Após consultar este médico percebi muito melhor a minha doença. & $\square$ & $\square$ & $\square$ & $\square$ & $\square$ \\
\hline $\begin{array}{l}\text { 13. O médico interessou-se por mim como pessoa e não somente } \\
\text { pela doença. }\end{array}$ & $\square$ & $\square$ & $\square$ & $\square$ & $\square$ \\
\hline 14. Este médico sabe tudo a meu respeito. & $\square$ & $\square$ & $\square$ & $\square$ & $\square$ \\
\hline 15. Senti que este médico percebia exactamente o que eu pensava. & $\square$ & $\square$ & $\square$ & $\square$ & $\square$ \\
\hline 16. Gostaria de ter tido um pouco mais de tempo de consulta. & $\square$ & $\square$ & $\square$ & $\square$ & $\square$ \\
\hline 17. Não fiquei totalmente satisfeito com a consulta. & $\square$ & $\square$ & $\square$ & $\square$ & $\square$ \\
\hline 18. Seria difícil contar ao médico aspectos muito pesso & $\square$ & $\square$ & $\square$ & $\square$ & $\square$ \\
\hline
\end{tabular}




\section{QUESTIONÁRIO FINAL}

Finalmente, gostaríamos de saber o que o médico fez, ou não, nesta consulta.

Nesta consulta o meu médico...

Sim

Não

1. ... fez-me uma observação física (por exemplo: auscultar, ver a garganta, palpar a barriga, medir a tensão arterial, etc.).

2. ... pediu-me exames (análises, radiografias, electrocardiograma, etc.).

3. ... enviou-me para uma consulta de outra especialidade (cirurgia, cardiologia, fisioterapia, etc.).

4. ... passou-me a receita para um novo medicamento (medicamento que não tomava habitualmente).

5. ... passou-me um papel / documento (atestado, baixa, declaração, etc.).

6. ... explicou-me a minha doença / as minhas queixas.

7. ... explicou-me o que observou em mim (auscultação, garganta, barriga, tensão arterial, etc.).

8. ... explicou-me os resultados dos exames.

9. ... explicou-me como vai ser o meu tratamento (como tomar os medicamentos, outros tratamentos, consultas de seguimento, etc.).

10. ... deu-me conselhos sobre como viver saudavelmente (alimentação, exercício, stress, etc.).

\section{Obrigado pela sua colaboração!}

